\title{
Effect of COPD treatments on MRPI-mediated transport in bronchial epithelial cells
}

\author{
Margaretha van der Deen' \\ Sandra Homan' \\ Hetty Timmer-Bosscha' \\ Rik J Scheper ${ }^{2}$ \\ Wim Timens ${ }^{3}$ \\ Dirkje S Postma ${ }^{4}$ \\ Elisabeth G de Vries' \\ Departments of 'Medical Oncology, \\ ${ }^{3}$ Pathology, ${ }^{4}$ Pulmonary Diseases, \\ University Medical Center Groningen \\ and University of Groningen, \\ The Netherlands; ${ }^{2}$ Department \\ of Pathology, VU University Medical \\ Center, Amsterdam, The Netherlands
}

\begin{abstract}
Background: Smoking is the principle risk factor for development of chronic obstructive pulmonary disease (COPD). Multidrug resistance-associated protein 1 (MRP1) is known to protect against toxic compounds and oxidative stress, and might play a role in protection against smoke-induced disease progression. We questioned whether MRP1-mediated transport is influenced by pulmonary drugs that are commonly prescribed in COPD.
\end{abstract}

Methods: The immortalized human bronchial epithelial cell line $16 \mathrm{HBE} 14 \mathrm{o}^{-}$was used to analyze direct in vitro effects of budesonide, formoterol, ipratropium bromide and $\mathrm{N}$-acetylcysteine (NAC) on MRP1-mediated transport. Carboxyfluorescein (CF) was used as a model MRP1 substrate and was measured with functional flow cytometry.

Results: Formoterol had a minor effect, whereas budesonide concentration-dependently decreased CF transport by MRP1. Remarkably, addition of formoterol to the highest concentration of budesonide increased CF transport. Ipratropium bromide inhibited CF transport at low concentrations and tended to increase $\mathrm{CF}$ transport at higher levels. NAC increased CF transport by MRP1 in a concentration-dependent manner.

Conclusions: Our data suggest that, besides their positive effects on respiratory symptoms, budesonide, formoterol, ipratropium bromide, and NAC modulate MRP1 activity in bronchial epithelial cells. Further studies are required to assess whether stimulation of MRP1 activity is beneficial for long-term treatment of COPD.

Keywords: bronchus epithelium, COPD, drugs, MRP1, multidrug resistance, oxidative stress

\section{Background}

Smoking generates oxidative stress in the lungs and is the principal risk factor for development of lung cancer and chronic obstructive pulmonary disease (COPD). Detoxification and elimination processes of noxious substances present in cigarette smoke are important for both disease prevention and progression, yet little is known on these processes in the lung so far. Proteins of the ATP-binding cassette (ABC) superfamily such as the multidrug resistance-associated protein 1 (MRP1) may play a role, since they protect against oxidative stress and other xenobiotics (Cole et al 1992). Substrates for MRP1 are glutathione, glucuronate, and sulfate conjugates and unconjugated compounds in presence of glutathione, eg, tobacco-specific nitrosamines (Leslie et al 2001). Interestingly, the lung and trachea and other tissues with a barrier function highly express several ABC transporters (Langmann et al 2003). Especially MRP1 is expressed at high levels in human lung tissue (van der Deen et al 2005), mainly at the basolateral side of bronchial epithelium (Brechot et al 1998; Scheffer et al 2002). We observed that MRP1 expression is diminished in bronchial epithelial cells of COPD patients (van der Deen et al 2006) supporting the hypothesis that lower functional MRP1 activity is related to COPD development.

So far, COPD is recognized as a relentlessly progressive disease in which only smoking cessation reduces the accelerated lung function decline. There is no 
cure for COPD, yet recently it has been shown that some drugs are beneficial in the disease management. Inhaled corticosteroids and long-acting beta-agonists such as budesonide and formoterol reduce the number of exacerbations in COPD (Alsaeedi et al 2002; Sutherland et al 2003) and their combination has been shown to be very effective (Calverley et al 2003). Treatment of COPD patients with the anticholinergic drug ipratropium bromide results in a small improvement of lung function, yet does not influence the long-term decline in mild COPD (Anthonisen et al 1994). Studies on oral use of the anti-mucolytic drug $\mathrm{N}$-acetylcysteine (NAC) have provided contradictory results (Stey et al 2000; Decramer et al 2005).

In contrast to the extensive knowledge on chemotherapeutic drugs as substrates for MRP1, limited data are available on the effect of pulmonary drugs on the functional expression of MRP1 (Hamilton et al 2002). NAC induces higher cellular glutathione levels and in this way can protect against oxidative stress that may indirectly affect MRP1 function (Akan et al 2005) but no information is available about budesonide, formoterol, and ipratropium bromide in this respect.

In the present study, we questioned whether medications commonly prescribed to COPD patients affect MRP1mediated transport. Therefore, we analyzed the direct in vitro effects of budesonide, formoterol, ipratropium bromide, and NAC on MRP1 by means of functional flow cytometry in immortalized human bronchial epithelial cells.

\section{Methods}

\section{Chemicals, media, and reagents}

Bovine serum albumin (BSA, fraction V), minimal essential medium (MEM, supplemented with Earle's salts and L-glutamine) and RPMI 1640 medium (supplemented with $25 \mathrm{mM}$ Hepes and L-glutamine) were purchased from Invitrogen Life Technologies (Breda, The Netherlands). Carboxyfluorescein diacetate (CFDA), ipratropium bromide, NAC and propidium iodide (PI) were obtained from Sigma-Aldrich BV (Zwijndrecht, The Netherlands). Budesonide (Pulmicort) was obtained from AstraZeneca BV (Zoetermeer, The Netherlands), formoterol fumarate dihydrate from Astra Draco AB (Lund, Sweden), ethylenedinitrilo tetraacetic acid disodiumsalt dihydrate (EDTA) from Merck (Darmstadt, Germany), fetal calf serum (FCS) from Bodinco BV (Alkmaar, The Netherlands), Vitrogen from Nutacon (Leimuiden, The Netherlands), and MK571 from Omnilabo (Breda, The Netherlands).

\section{Bronchial cells}

The human bronchial epithelial cell line 16HBE140*, immortalized with pSVori plasmid transfection, was kindly provided by Dr. DC Gruenert (California Pacific Medical Center Research institute; San Francisco, CA) (Cozens et al 1994). This cell line expresses MRP1 at relatively moderate levels. Cells were cultured in MEM supplemented with $10 \%$ heat inactivated FCS. Before trypsinization, cells were washed twice with phosphate buffered saline (PBS: $6.4 \mathrm{mM}$ Na2HPO4, 1.5 mM KH2PO4, 0.14 mM NaCl, 2.7 mM KCl, $\mathrm{pH}=7.4$ ) with $0.5 \mathrm{mM}$ EDTA. $16 \mathrm{HBE} 14 \mathrm{o}^{-}$cells were grown on tissue culture plastics coated with Vitrogen $(30 \mu \mathrm{g} / \mathrm{mL})$ and BSA $(10 \mu \mathrm{g} / \mathrm{mL})$.

\section{Flow cytometry}

To determine MRP1-mediated transport, cells were incubated with $0.1 \mu \mathrm{M}$ CFDA as described previously (van der Kolk et al 1998) with slight modifications. CFDA is intracellularly converted to carboxyfluorescein (CF) which is a fluorescent MRP1 substrate. To establish the effect of COPD drugs on MRP1-mediated activity, $1 \times 10^{6}$ cells were incubated in $0.5 \mathrm{~mL}$ RPMI 1640 medium without FCS $\left(37^{\circ} \mathrm{C}, 5 \% \mathrm{CO}_{2}\right)$ with CFDA and with or without the addition of drugs for 1 hour. MK571 (20 $\mu \mathrm{M})$ was used as a positive control for inhibition of MRP1 activity (van der Kolk et al 1998) and always showed strong inhibition (more than 10-fold) of MRP1-mediated efflux of CF. Budesonide and formoterol were added in concentrations of $10^{-8} \mathrm{M}$ to $10^{-4} \mathrm{M}$. These drugs were dissolved in $96 \%$ ethanol and dimethyl sulfoxide (DMSO), respectively. In part of the experiments, cells were simultaneously incubated with budesonide and formoterol. When budesonide and formoterol are combined in one inhaler (eg, Symbicort ${ }^{\circledR}$, AstraZeneca), the budesonide concentration is $\sim 18$ - to 35 -fold higher than the concentration of formoterol; therefore we added concentrations of either drug with a comparable proportion. Ipratropium bromide was added in concentrations from $10^{-7} \mathrm{M}$ to $2 \times 10^{-4} \mathrm{M}$ and NAC from $10^{-4} \mathrm{M}$ to $1.6 \times 10^{-3} \mathrm{M}$ and these drugs were dissolved in PBS and water respectively.

Cells were pelleted for 15 seconds at 12,000 $\mathrm{g}$ and resuspended in ice-cold RPMI medium without FCS. Drug or MK571 was added a second time in appropriate concentrations for 1 hour, this time without substrate $\mathrm{CFDA}\left(37^{\circ} \mathrm{C}\right.$, $5 \% \mathrm{CO}_{2}$ ). After pelleting, cells were put on ice to stop efflux of substrate and were resuspended in $350 \mu \mathrm{L}$ RPMI medium with $0.1 \mu \mathrm{g} / \mathrm{mL}$ PI to distinguish dead from living cells.

Fluorescence of CF was analyzed with a FACSCalibur ${ }^{\mathrm{TM}}$ flow cytometer (Becton Dickinson Medical Systems; 
Franklin Lakes, NY, USA). We measured 10,000 events per sample (living cells). The Winlist 5.1 program (Verity Software House Inc.; Topsham, ME, USA) was used to calculate mean fluorescence intensity (MFI) values. All measurements were corrected for the negative control (medium alone) and for incubation with the solvents $96 \%$ ethanol and DMSO if appropriate. The percentage of dead cells (PI positive population) did not increase with all tested drug concentrations. None of the tested drugs caused autofluorescence (fluorescence without CFDA and PI incubation).

\section{Statistical analysis}

Data are expressed as the mean \pm standard error of the mean (SEM). All means were calculated from 3 to 6 independent experiments. The paired Student's t-test (two-tailed) was used to calculate modulating effects of the drug under study compared to control. Differences were considered significant when $\mathrm{p}<0.05$. Statistical analyses were performed with SPSS 12 (SPSS Inc.; Chicago, IL).

\section{Results}

\section{Modulating effect of budesonide and formoterol on MRPI-mediated activity}

Budesonide increased the accumulation of $\mathrm{CF}$ at concentrations of $10^{-5} \mathrm{M}$ and $10^{-4} \mathrm{M}$ with $26 \%$ and $97 \%$, respectively. Lower concentrations did not affect $\mathrm{CF}$ accumulation significantly (Figure 1A). Formoterol had a small yet significant

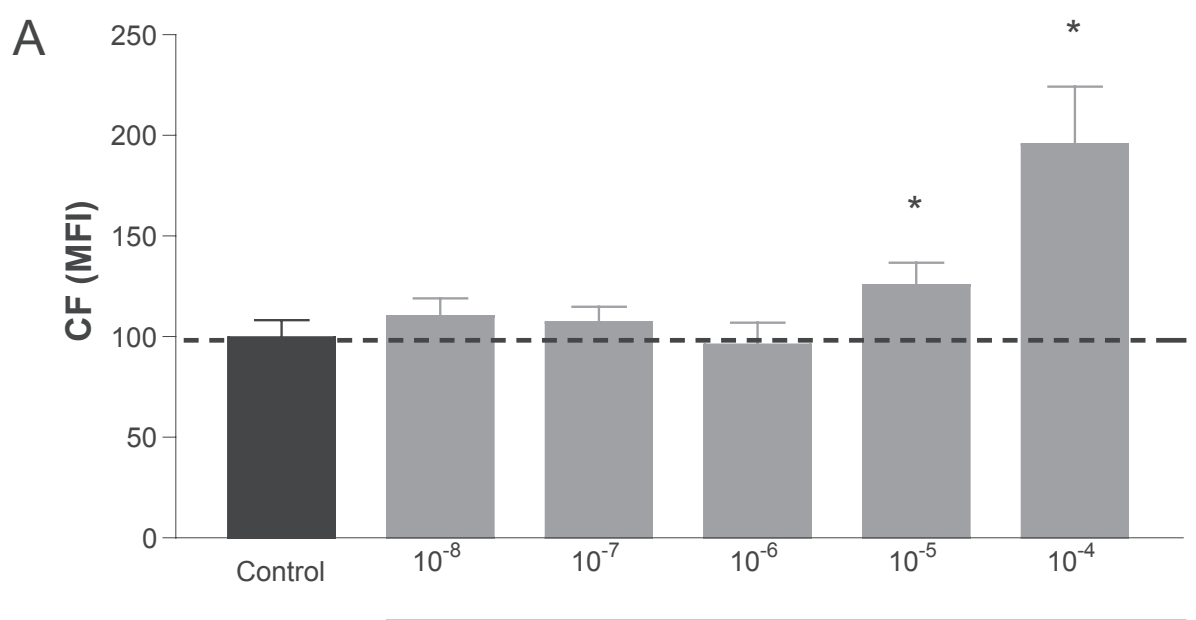

Budesonide (M)

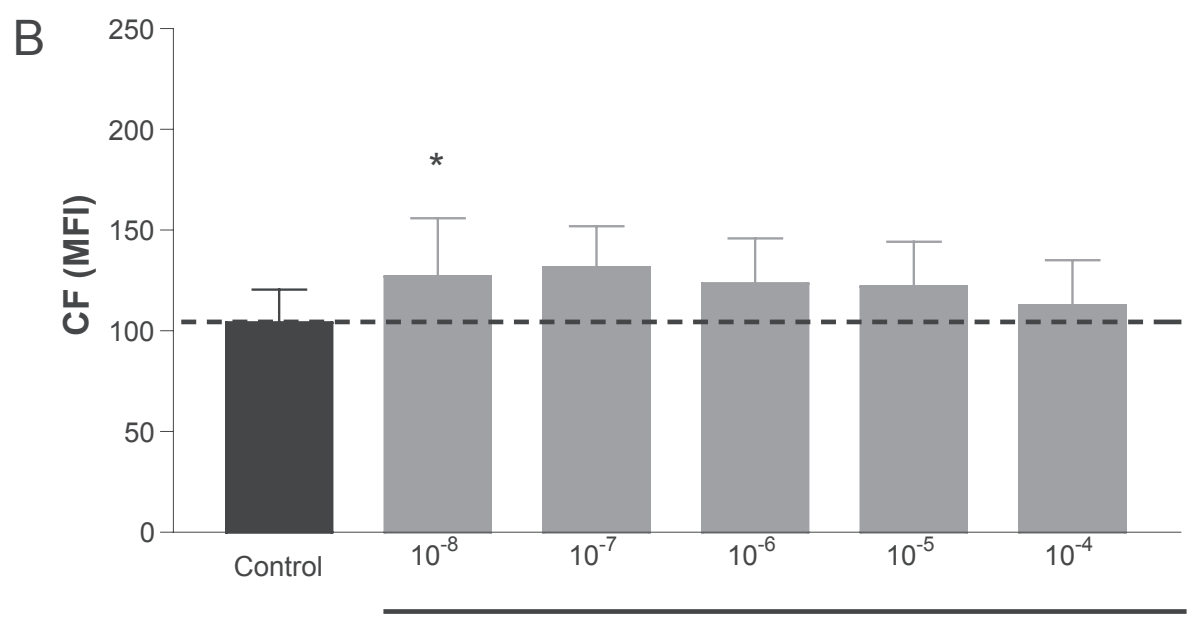

Formoterol (M)

Figure I Modulation of MRPI-mediated activity with increasing concentrations of (A) budesonide or (B) formoterol. Dashed line indicates the MFI level of the control (incubation with vehicle). Data are shown of mean \pm SEM of 3-4 independent experiments for budesonide and $5-6$ independent experiments for formoterol. *P $<0.05$ compared with control.

Abbreviations: CF, carboxyfluorescein; MFI, mean fluorescence intensity; MRPI, multidrug resistance-associated protein I. 
effect on the accumulation of $\mathrm{CF}$ at $10^{-8} \mathrm{M}$, but at higher concentrations this effect was not significant (Figure 1B).

COPD patients are often treated with a combined therapy of budesonide and formoterol. To address the question whether the effect of budesonide is altered by addition of formoterol, we incubated the highest concentration of budesonide $\left(10^{-4} \mathrm{M}\right)$ with increasing concentrations of formoterol. With this approach, the budesonide-induced CF accumulation decreased in a dose-dependent manner; with $10^{-6} \mathrm{M}, 10^{-5} \mathrm{M}$, and $10^{-4} \mathrm{M}$ formoterol, respectively, $198 \%$, $124 \%$, and $58 \%$ compared with the control (Figure 2).

\section{Modulating effect of ipratropium bromide and $\mathrm{N}$-acetylcysteine on MRPI-mediated activity}

Ipratropium bromide increased $\mathrm{CF}$ accumulation at relatively low concentrations $\left(10^{-7} \mathrm{M}\right.$ to $\left.10^{-5} \mathrm{M}\right)$ with a maximum of $24 \%$ at $10^{-7} \mathrm{M}$ (Figure $3 \mathrm{~A}$ ). Increasing the concentration of ipratropium bromide to $2 \times 10^{-4} \mathrm{M}$ potentiated the $\mathrm{CF}$ efflux $(56 \%$ compared with control), suggesting that MRP1-mediated activity is stimulated rather than inhibited with ipratropium bromide. Similar results were obtained with NAC, ie, CF accumulation was reduced in a concentration-dependent manner $(57 \%$ at $8 \times$ $10^{-4} \mathrm{M}$ ) (Figure 3B). Increasing the NAC concentrations to $1.6 \times 10^{-3} \mathrm{M}$ potentiated the $\mathrm{CF}$ efflux even more.

\section{Discussion}

The membrane protein MRP1 is highly expressed in human lung tissue and may protect the airways against damage induced by cigarette smoking (Scheffer et al 2002). This study shows for the first time the direct influence of pulmonary drugs on MRP1 functional activity, drugs that have already proven their effectiveness in the clinical management of COPD.

Since MRP1 function is most likely cytoprotective in lung cells, we argued that modulation of its function with pulmonary drugs might induce either a positive or negative effect in COPD with long-term treatment. We observed that budesonide interfered with MRP1-mediated transport by increasing CF retention. This implies either that budesonide is an MRP1 substrate or that it acts on MRP1 function in another way. Budesonide is intracellularly esterified to a fatty acid which is thought to be the mechanism of its prolonged activity after a single dose (O'Connell 2003). MRP1 has been shown to be capable of rather slow outward transport of phospholipids, phophatidylcholine and sphingomyelin, and phospholipid analogues in the presence of oxidized glutathione and ATP in human erythrocytes and epithelial cells (Dekkers et al 2000). It is tempting to speculate that esterified budesonide could behave like a phospholipid analogue with a polar head and two fatty acid tails and is then outwards transported by MRP1. As such, budesonide can compete with other MRP1 (physiological) substrates and in that way affect the functional activity of MRP1.

We measured the direct effect of budesonide on MRP1-mediated transport. In addition, budesonide is also able to affect the transcriptional regulation of MRP1, as shown by diminished expression of MRP1 in the lung epithelial cell line Calu-1 after long-term incubation with

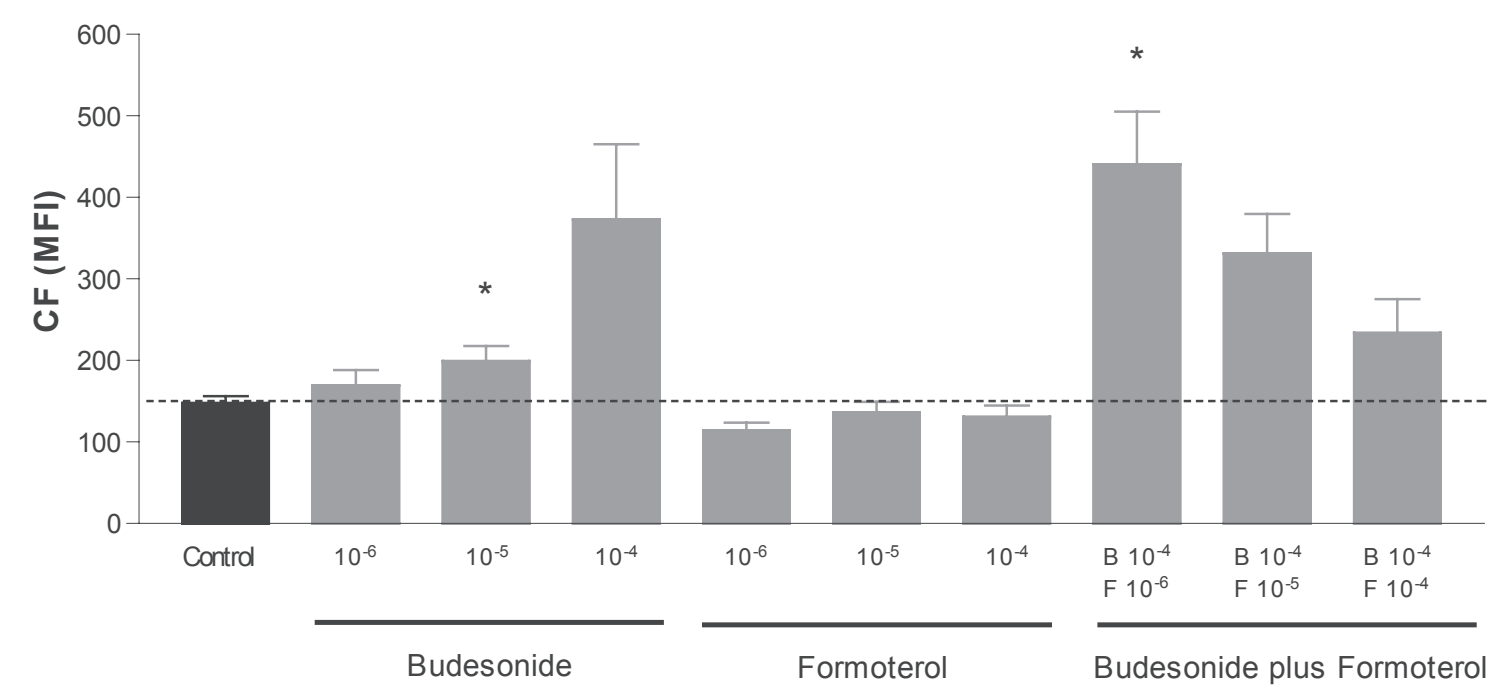

Figure 2 Modulation of MRPI activity by budesonide, formoterol, or their combination. Dashed line indicates the MFI level of the control (incubation with vehicle). Data are shown of mean \pm SEM of 3 independent experiments. *p $<0.05$ compared with control.

Abbreviations: CF, carboxyfluorescein; MFI, mean fluorescence intensity; MRPI, multidrug resistance-associated protein I. 


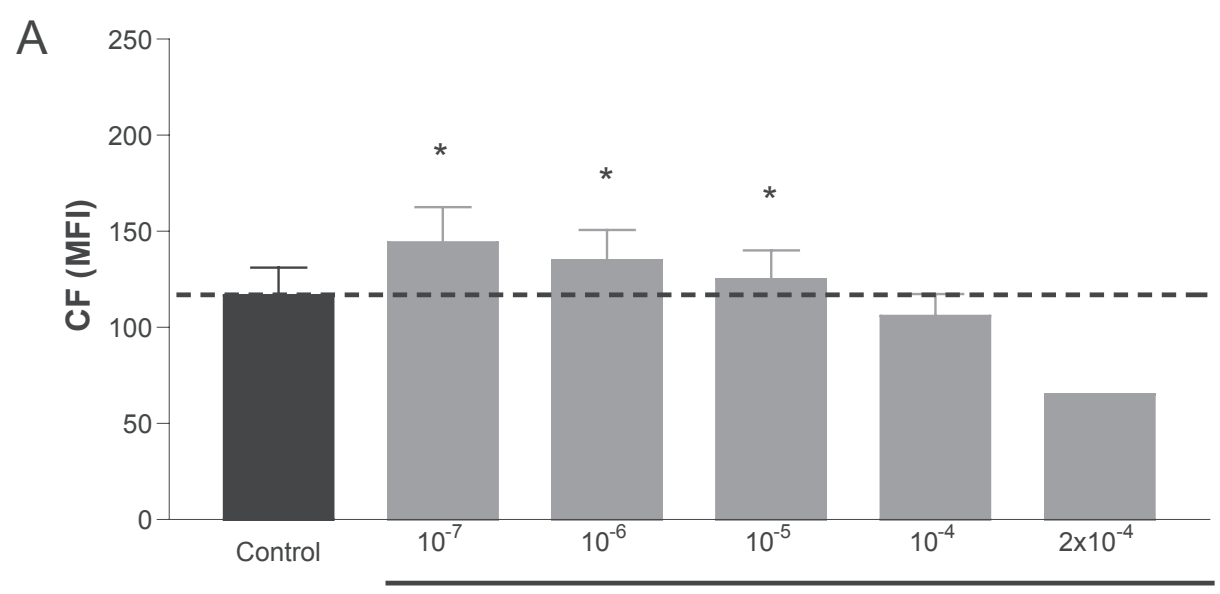

Ipratropium bromide $(\mathrm{M})$

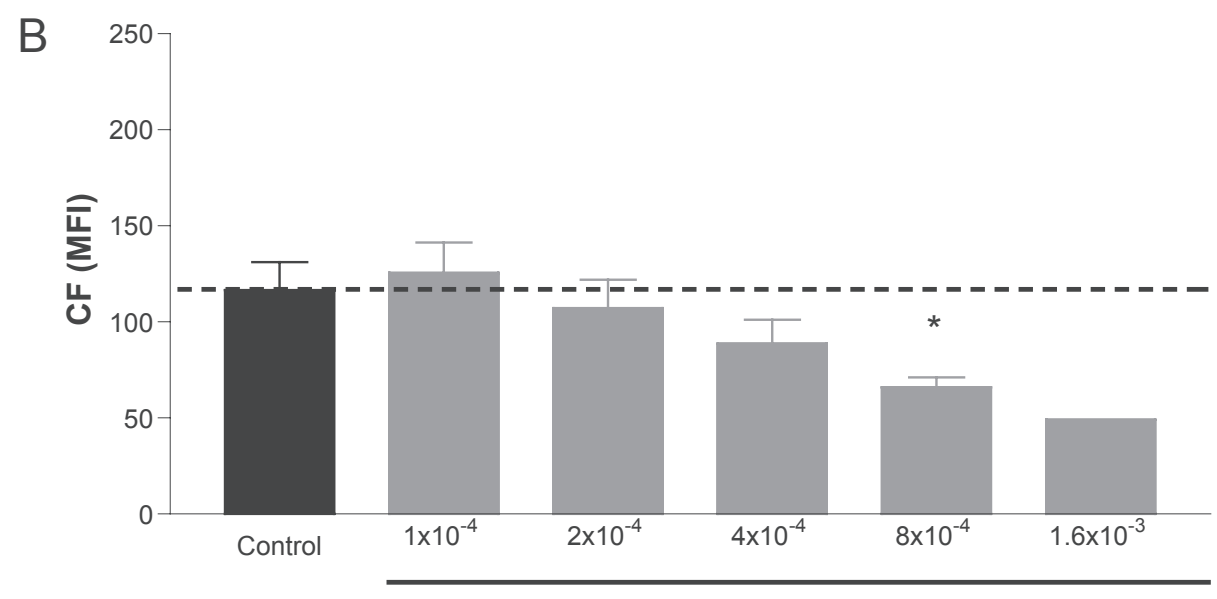

$\mathrm{N}$-acetylcysteine $(\mathrm{M})$

Figure 3 Modulation of MRPI-mediated activity with increasing concentrations of (A) ipratropium bromide or (B) N-acetylcysteine. Dashed line indicates the MFI level of the control (incubation with vehicle). Data are shown of mean $\pm S E M$ of independent experiments for ipratropium bromide $\left(n=5\right.$ for $10^{-7}$ to $10^{-4} M ; n=I$ for $\left.2 \times 10^{-4} M\right)$, and for $\mathrm{N}$-acetylcysteine $\left(\mathrm{n}=3-6\right.$ for $\mathrm{I} \times 10^{-4}$ to $8 \times 10^{-4} \mathrm{M} ; \mathrm{n}=\mathrm{I}$ for $\left.1.6 \times 10^{-3} \mathrm{M}\right)$. ${ }^{*} \mathrm{p}<0.05$ compared with control.

Abbreviations: CF, carboxyfluorescein; MFI, mean fluorescence intensity; MRPI, multidrug resistance-associated protein I.

budesonide $\left(10^{-5} \mathrm{M}\right)$ (Bandi and Kompella 2002). Taken together, the data suggest that treatment with budesonide could potentially have a negative effect in COPD, given the cell protective properties of MRP1. Our observation does not distract from the well known beneficial effects of budesonide in diseases like asthma, since steroids suppress virtually every step of the inflammatory cascade. However, our findings may contribute an alternative hypothesis why inhaled steroids are not capable of altering the long-term course of COPD, particularly in smokers with COPD (Alsaeedi et al 2002).

In combination with formoterol, the direct inhibitory effect of budesonide on $\mathrm{CF}$ accumulation of epithelial cells was reduced, whereas formoterol as such had only minor effects on MRP1-mediated transport. This indicates that budesonide or its esterified derivatives is less available as an MRP1 substrate/inhibitor when combined with formoterol or that formoterol interferes with the interaction between budesonide (esters) and MRP1. Although formoterol is moderately lipophilic, it has a high affinity for cellular membrane lipid bilayers (Anderson et al 1994). High concentrations of formoterol might interfere with the function of membrane proteins as this depends on the lipid environment of the protein. The effect with formoterol may also indirectly run via adenosine 3',5'-cyclic monophosphate (cAMP) since formoterol stimulates cells via the beta(2)adrenergic receptor (Johnson and Rennard 2001) resulting in higher intracellular levels of cAMP. However, it is not clear why this occurs in the presence of budesonide while formoterol alone had no effect.

Ipratropium bromide incubation resulted in lower CF retentions with increasing concentrations in a 
concentration-dependent manner. Ipratropium bromide competitively inhibits acetylcholine binding to the muscarinic receptor resulting in decreased guanosine 3',5'-cyclic monophosphate (cGMP) levels which lowers bronchoconstriction. There are no reports in the literature on its mechanism of action with respect to MRP1, but it can be speculated that cGMP might play a role in this process since cGMP is a substrate of other members of the MRP family, MRP4 and MRP5 (Wielinga et al 2003).

Effects of NAC were similar to those observed with ipratropium bromide, ie, MRP1-mediated transport was stimulated with increasing concentrations of NAC. This may be due to the fact that NAC elevates glutathione levels. Glutathione and many glutathione conjugates are substrates for MRP1, and therefore, intracellular glutathione levels are of major importance for MRP1 function in antioxidant defense. It is known that transport of anionic species such as CF can be stimulated with glutathione (Akan et al 2005; Bagrij et al 2001). It was observed that the compounds sulfinpyrazone and indomethacin stimulate glutathione transport by MRP2 at low concentrations, whereas relatively high concentrations inhibit GSH transport into the medium (Evers et al 2000). A possible explanation was given that both compounds are transported cooperatively. Our results with NAC and ipratropium bromide might be explained by a similar co-transport of these substances with CF. It may occur that certain (at present undefined) toxic inhaled substances can be transported by MRP1 more effectively with increasing glutathione levels as well. For example, the tobacco-derived NNAL-O-glucuronide was identified as an MRP1 substrate that requires glutathione for transport (Leslie et al 2001).

The pulmonary drugs that we tested are, apart from NAC, clinically administered by inhalation. These drugs act directly on epithelial cells or pass the epithelial layer and act on eg smooth muscle cells or inflammatory cells. Clinically achievable concentrations at the level of airway epithelium are mainly unknown. The intracellular levels that can be reached with treatment highly depend on the biochemical features of the substance, action of drug metabolism proteins (phase II conjugation enzymes such as cytochrome P450 isoforms), drug efflux pumps (phase III systems), as well as the route of administration. We have chosen the ranges of the applied drug concentrations based on published data (Gustafsson and Persson 1991; Anderson et al 1994; Gillissen et al 1997; Nagy et al 1997; Bandi and Kompella 2002; Borchard et al 2002) and theoretical models, ie, ranges that will be clinically effective. MRP1 is located in bronchial epithelium, the first cells that drugs have to encounter to reach the underlying tissue to act on eg smooth muscle cells, fibroblasts and inflammatory cells. Thus, local high cellular concentrations of pulmonary drugs are very likely to affect MRP1 activity in bronchial epithelium which was indeed confirmed in vitro in the present study.

In conclusion, our studies show that drugs that are clinically used in COPD affect MRP1 function, a transporter that protects against oxidative stress and toxic compounds. Budesonide inhibits this MRP1-mediated transport and addition of formoterol on its turn reduces this inhibitory effect whereas ipratropium bromide and NAC stimulate MRP1 activity in a concentration-dependent manner. Further studies are required to investigate whether stimulation of MRP1 activity is beneficial for long-term treatment of COPD with regard to the protective properties of MRP1.

\section{Acknowledgments}

This work was supported by a grant from the Netherlands Asthma Foundation (NAF97.35) and "Stichting Astma Bestrijding" (SAB), The Netherlands.

\section{Disclosures}

None of the authors has any conflicts of interest to disclose.

\section{References}

Akan I, Akan S, Akca H, et al. 2005. Multidrug Resistance-Associated Protein 1 (MRP1) mediated vincristine resistance: effects of N-acetylcysteine and buthionine sulfoximine. Cancer Cell Int, 5:22.

Alsaeedi A, Sin DD, McAlister FA. 2002. The effects of inhaled corticosteroids in chronic obstructive pulmonary disease: a systematic review of randomized placebo-controlled trials. Am J Med, 113:59-65.

Anderson GP, Linden A, Rabe KF. 1994. Why are long-acting beta-adrenoceptor agonists long-acting? Eur Respir J, 7:569-78.

Anthonisen NR, Connett JE, Kiley JP, et al. 1994. Effects of smoking intervention and the use of an inhaled anticholinergic bronchodilator on the rate of decline of FEV1. The Lung Health Study. JAMA, 272:1497-505.

Bagrij T, Klokouzas A, Hladky SB, et al. 2001. Influences of glutathione on anionic substrate efflux in tumour cells expressing the multidrug resistanceassociated protein, MRP1. Biochem Pharmacol, 62:199-206.

Bandi N, Kompella UB. 2002. Budesonide reduces multidrug resistanceassociated protein 1 expression in an airway epithelial cell line (Calu-1). Eur J Pharmacol, 437:9-17.

Borchard G, Cassara ML, Roemele PE, et al. 2002. Transport and local metabolism of budesonide and fluticasone propionate in a human bronchial epithelial cell line (Calu-3). J Pharm Sci, 91:1561-7.

Brechot JM, Hurbain I, Fajac A, et al. 1998. Different pattern of MRP localization in ciliated and basal cells from human bronchial epithelium. J Histochem Cytochem, 46:513-7.

Calverley PM, Boonsawat W, Cseke Z, et al. 2003. Maintenance therapy with budesonide and formoterol in chronic obstructive pulmonary disease. Eur Respir J, 22:912-9.

Cole SP, Bhardwaj G, Gerlach JH, et al. 1992. Overexpression of a transporter gene in a multidrug-resistant human lung cancer cell line. Science, 258:1650-4. 
Cozens AL, Yezzi MJ, Kunzelmann K, et al. 1994. CFTR expression and chloride secretion in polarized immortal human bronchial epithelial cells. Am J Respir Cell Mol Biol, 10:38-47.

Decramer M, Rutten-van Molken M, Dekhuijzen PN, et al. 2005. Effects of $\mathrm{N}$-acetylcysteine on outcomes in chronic obstructive pulmonary disease (Bronchitis Randomized on NAC Cost-Utility Study, BRONCUS): a randomised placebo-controlled trial. Lancet, 365:1552-60.

Dekkers DW, Comfurius P, van Gool RG, et al. 2000. Multidrug resistance protein 1 regulates lipid asymmetry in erythrocyte membranes. Biochem $J, 350: 531-5$.

Evers R, de Haas M, Sparidans R, et al. 2000. Vinblastine and sulfinpyrazone export by the multidrug resistance protein MRP2 is associated with glutathione export. Br J Cancer, 83:375-83.

Gillissen A, Jaworska M, Orth M, et al. 1997. Nacystelyn, a novel lysine salt of $\mathrm{N}$-acetylcysteine, to augment cellular antioxidant defence in vitro. Respir Med, 91:159-68.

Gustafsson B, Persson CG. 1991. Effect of different bronchodilators on airway smooth muscle responsiveness to contractile agents. Thorax, 46:360-5.

Hamilton KO, Yazdanian MA, Audus KL. 2002. Contribution of efflux pump activity to the delivery of pulmonary therapeutics. Curr Drug Metab, 3:1-12.

Johnson M, Rennard S. 2001. Alternative mechanisms for long-acting beta(2)-adrenergic agonists in COPD. Chest, 120:258-70.

Langmann T, Mauerer R, Zahn A, et al. 2003. Real-time reverse transcription-PCR expression profiling of the complete human ATPbinding cassette transporter superfamily in various tissues. Clin Chem, 49:230-8.

Leslie EM, Deeley RG, Cole SP. 2001. Toxicological relevance of the multidrug resistance protein 1, MRP1 (ABCC1) and related transporters. Toxicology, 167:3-23.
Leslie EM, Ito K, Upadhyaya P, et al. 2001. Transport of the beta$\mathrm{O}$-glucuronide conjugate of the tobacco-specific carcinogen 4-(methylnitrosamino)-1-(3-pyridyl)-1-butanol (NNAL) by the multidrug resistance protein 1 (MRP1). Requirement for glutathione or a non-sulfur-containing analog. $J$ Biol Chem, 276:27846-54.

Nagy AM, Vanderbist F, Parij N, et al. 1997. Effect of the mucoactive drug nacystelyn on the respiratory burst of human blood polymorphonuclear neutrophils. Pulm Pharmacol Ther, 10:287-92.

O'Connell EJ. 2003. Review of the unique properties of budesonide. Clin Ther, 25(Suppl C):C42-C60.

Scheffer GL, Pijnenborg AC, Smit EF, et al. 2002. Multidrug resistance related molecules in human and murine lung. J Clin Pathol, 55:332-9.

Stey C, Steurer J, Bachmann S, et al. 2000. The effect of oral N-acetylcysteine in chronic bronchitis: a quantitative systematic review. Eur Respir $J$, 16:253-62.

Sutherland ER, Allmers H, Ayas NT, et al. 2003. Inhaled corticosteroids reduce the progression of airflow limitation in chronic obstructive pulmonary disease: a meta-analysis. Thorax, 58:937-41.

van der Deen M, de Vries EG, Timens W, et al. 2005. ATP-binding cassette (ABC) transporters in normal and pathological lung. Respir Res, 6:59.

van der Deen M, Marks H, Willemse BW, et al. 2006. Diminished expression of multidrug resistance-associated protein 1 (MRP1) in bronchial epithelium of COPD patients. Virchows Arch, 449:682-8.

van der Kolk DM, de Vries EG, Koning JA, et al. 1998. Activity and expression of the multidrug resistance proteins MRP1 and MRP2 in acute myeloid leukemia cells, tumor cell lines, and normal hematopoietic CD34+ peripheral blood cells. Clin Cancer Res, 4:1727-36.

Wielinga PR, van der Heijden I, Reid G, et al. 2003. Characterization of the MRP4- and MRP5-mediated transport of cyclic nucleotides from intact cells. J Biol Chem, 278:17664-71. 
